\title{
Maternal near miss reviews in tertiary care centres - a real necessity
}

\author{
Prof (Dr) Hem Kanta Sarma \\ Professor \& HOD, Obstetrics\& Gynaecology, Jorhat Medical College, Jorhat, India. \\ Correspondence: Prof (Dr) Hem Kanta Sarma, Professor \& HOD, Obstetrics\& Gynaecology, \\ Jorhat Medical College, Jorhat, India; Email- sarmahemkanta@gmail.com
}

Distributed under Creative Commons Attribution-Share Alike 4.0 International.

More than $90 \%$ of the maternal deaths in India, more so in north eastern India including Assam are preventable. These are almost always preceded by severe acute maternal morbidity or maternal near miss morbidity. Reviews of maternal mortality and near miss maternal morbidity are of paramount importance in an effort of continuous improvement of maternal health care of a community. These reviews will expose different types of factors, but they are complementary to each other. Reviews and studies of severe acute maternal morbidity always boost in the effort of preventing maternal mortality and improve the obstetric care of the community [1].

Near miss cases during pregnancy represents part of a continuum between extreme of good health and death (figure-1). In this stage organ dysfunction or failure occurs. Timely and judicious medical intervention at this stage may save the patient and avert a maternal death. As the modern science is advancing there is more and more reduction in maternal mortality. This has increased the importance of near miss maternal morbidity for further improvement in the health care system. Prevention of near miss cases is possible by implementing effective essential obstetric care. Management of near miss cases is possible by timely intervention by emergency obstetric care.

In 2007 the World Health Organization (WHO) established a technical working group comprising obstetricians, midwives, epidemiologists, and public health care professionals to develop a standard definition and formulate identification criteria for maternal near miss cases. The WHO near miss approach is best implemented in 3 steps - 1) Baseline assessment, 2) Situation analysis and 3) Intervention for improving health care.

Maternal near miss case is defined as a woman who nearly died but survived a complication that occurred during pregnancy, childbirth or within 42 days of termination of pregnancy [2]. The World Health Organization defines direct obstetric morbidity as resulting from obstetric complications of the pregnancy states from interventions, omissions, incorrect treatment, or from a chain of events resulting from any of the above [3]. The inclusion criteria for maternal near miss are grouped in 3 categories: 1) Clinical, 2) Laboratory based and 3) Management based criteria. Currently the following criteria are accepted for identification of near miss cases: 1) Major obstetric haemorrhage, 2) Eclampsia, 3) Renal or Liver dysfunction, 4) Cardiac arrest, 5) Pulmonary oedema, 
6) Acute respiratory dysfunction, 7) Coma, 8) Cerebrovascular event, 9) Status epilepticus, 10) Anaphylactic shock, 11) Septicaemic shock, 12) Anaesthetic problem and 13) Massive pulmonary embolism.

Federation of Obstetrics and Gynaecological Societies of India (FOGSI) is currently working to develop a near miss registry with online reporting and to establish a national database on severe acute maternal morbidity to streamline the matter. Near miss cases are six fold higher than maternal deaths. It is easier to collect the data because unlike maternal death this does not involve the sense of guilt or fear. So it gives an opportunity for procuring good studies. Maternal near miss indicators are useful in analysing cause and effect of near miss cases as well as to formulate effective protocol for prevention and management of the cases. Some of the commonly used are: 1) Maternal near miss morbidity ratio (MNMR) Number of maternal near miss cases per 1000 live birth, 2) Severe maternal outcome ratio (SMOR) - It refers to the number of women with lifethreatening condition per 1000 live birth (SMOR = MNM $+\mathrm{MD} / \mathrm{LB}$ ). SMOR and MNMR indicates the amount of care and resources that would be needed in the area or facility level, 3) Maternal near miss mortality ratio ( MNM : MD ) - Higher ratio indicates better care at facility level and 4) Mortality index - MD/MNM+MD. Higher index indicates low quality care.

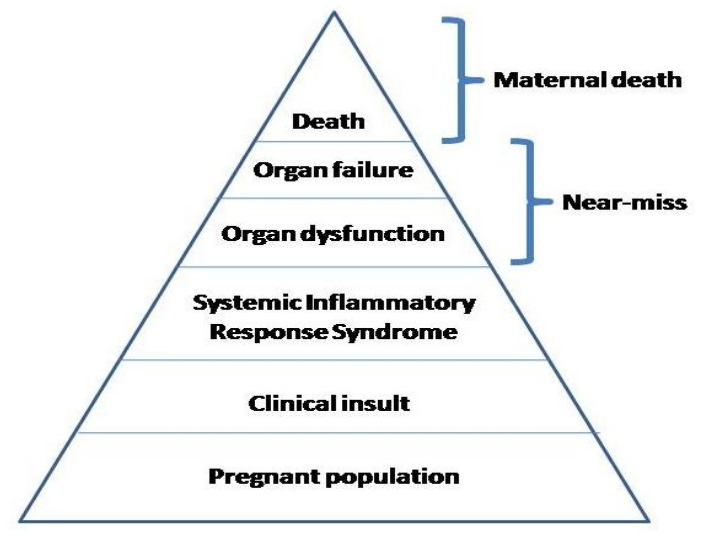

Figure 1. Maternal Health Pyramid
Very few studies are available in this topic in the north east India, a few studies are available in rest of India. Kalra $\mathrm{P}$ et al from Jodhpur, Rajasthan showed highest incidence of haemorrhage (56\%) followed by hypertension (17.8\%) [4]. Pandey A et al in a study of teaching hospital in north India found highest incidence of NMM in haemorrhage $(45.7 \%$ ) followed by hypertensive disorder (24.2\% ) [5]. Dev Sarma H K et al from Barpeta, Assam reported commonest cause of near miss as eclampsia (39.4\%) and also showed a $59 \%$ prevalence amongst primipara, with a alarmingly high incidence of $37.8 \%$ in the 15 to 20 years age group [6]. Maternal near miss ratio in Barpeta, Assam is 42.1/1000 live birth (Dev Sarma et al), Brazil is 21.3/1000 live birth, Jodhpur, Rajasthan 4/1000 live birth (Kalra $\mathrm{P}$ et al) and Kathmandu 23/1000 live birth. Maternal near miss mortality ratio in Kathmandu is 1: 6, while in Barpeta it is 1: 3.9 and in Rajasthan 1:2.07.

\section{Conclusion}

Maternal near miss audit is the need of the hour for all tertiary care centre including the medical colleges specially in the developing countries like India. This has not been a routine practice in all Indian teaching hospitals, more so in the north eastern part including Assam. Maternal near miss audit along with maternal death audit in combination will show us a new vista for logical improvement in the maternal health of the country. This is really a point of concern to note that even today in Barpeta, Assam we are loosing teen age mothers due to inadequate or inaccessible obstetric care and the social custom of child marriage with a horrible incidence of MNMR comprising a 37.8\% share.

\section{Conflict of interest: None. Disclaimer: Nil.}

\section{References}

1. Ramarajan A. Severe acute maternal morbidity: safe motherhood initiative - A silent revolution. New Delhi: Jaypee brothers medical publishers; 2014.

2. World Health Organization. Evaluating the quality of care for severe pregnancy complications. Geneva: Stationery Office; 2011. 
3. Measuring reproductive morbidity: report of a technical working group. Online $\mathrm{WHO} / \mathrm{MCH} / 90.4$ Unpublished 41p. Geneva: Stationary office; 1990.

4. KalraP, Kachhwaha P. Obstetric near miss morbidity and maternal mortality in a tertiary care centre in western Rajasthan. Indian Journal of Public health. 2014; 58 (3):199-201.

5. Pandey A, DasV, Agarwal A, Agrawal S, Misra D, Jaiswal N. Evaluation of obstetric near miss and maternal deaths in a tertiary care hospital in north India: shifting focus from mortality to morbidity. The Journal of obstetric and gynaecology of India. 2014; 64(6):394-99.

6. Dev Sarma HK, Sarma HK, Kalita AK. A prospective study of maternal near-miss and maternal mortality cases in FAAMCH, Barpeta; with special reference to its aetiology and management: First 4 months report. The Journal of Obstetrics and Gynaecology, Barpeta. 2014; 1(2): 100-106. 